\title{
Study of the Spatio-Temporal Parameters of the Gait of a Transtibial Amputee in Conditions of Alignment and Misalignment of the Prosthesis
}

\author{
Yuliana Ruiz, Brigette Torres, Esperanza Camargo \\ Universidad Distrital Francisco José de Caldas \\ Bogota, Colombia \\ yruizp@correo.udistrital.edu.co, bptorresb@correo.udistrital.edu.co, ecamargoc@udistrital.edu.co
}

\begin{abstract}
The study of the temporal spatial parameters of gait indicates in detail the biomechanical magnitudes that manifest in the gait cycle (velocities, lengths, times, cadence) in the joints involved.

Given the complexity of the study, the above parameters are studied from the perspective of alignment vs. the misalignment of a patient with a unilateral transtibial prosthesis in the sagittal and frontal plane with the base of the joint movements. This research evaluates the characteristics (magnitudes) of spontaneous velocity, steps length, stride time, cadence of steps taken from a Technaid® motion capture equipment (MOCAP), from the cinematography of joint movements in hip, knee and ankle, using the Human March software which delivers the temporal space variables. This analysis was performed in an orthopedic and rehabilitation center. This research and software have been developed by the research group DIGITI of the District University.
\end{abstract}

Keywords: Prosthetics, alignment, misalignment, spatio-temporal parameters, biomechanics

\section{Introduction}

The normal gait cycle in an amputee diverges in many ways: the extrinsic (terrain, footwear, development of the environment), intrinsic (sex, height, physical condition, anthropometry) and pathological factors of each patient differ in the gait cycle [1]. On the other hand, the design of each prosthesis: whether the type of amputation, the type of foot, the suspension, the mass, the prosthesis material and the angle of retraction is subjective. Additionally, the alignment of the prosthesis for each type of patient is different, since the gait process is a unique cycle.

The alignment of the prosthesis, understood as the relative position and orientation of a prosthesis, has three moments: the bench alignment where the prosthesis is assembled with the criteria prescribed by the specialist, the static alignment in which the position of the prosthesis in standing position and the dynamic alignment is observed evaluating the walking pattern. In all the previous ones the alignment is carried out in a subjective way, being subjected to the experience of the specialist. Although some research has been carried out on the use of technology for the evaluation of the effects of misalignment, these have been executed in gait laboratories, thus it is necessary the usage other and more accessible types of technologies given the increasing amputees population in Colombia, which reports 11,472 victims as a result of the armed conflict during 2016. [2]

This study incorporates the Technaid® IMU inertial sensor technology, since the equipment is portable and adjustable to any subject. The case study is a transtibial amputee in which the socket position was modified in the sagittal and frontal plane, alternating the alignment between 2 and 6 for flexion, extension, abduction and adduction. The results show evidences of alterations of the gait cycle and expose the correct position the prosthesis must have. This is done with a relationship of the patient with the prosthesis aligned vs. the misalignment conditions of the socket, obtaining an analysis of the temporal space parameters, such as: stride length, spontaneous speed, stride time, cadence and step time.

\section{Methods and Materials.}

To capture the movement, the Technaid ${ }^{\circ}$ equipment was implemented: The Tech MCS ${ }^{\circ}$ is a team that allows a complete analysis of the movement. Using an inertial sensor called Tech IMU, it is used in biomechanical research, 
rehabilitation and sports performance. The data output can be generated via USB port or Bluetooth communication with an indoor capture distance of $50 \mathrm{~m}$ and $150 \mathrm{~m}$ outdoors. It obtains data like: acceleration, angular velocity, magnetic field and articular angles. [3]

Each sensor was located at strategic sites: In the femur, tibia and foot, respectively for both limbs and the main sensor on the right lateral side of the patient.

The rectification of the data was done from the 3D avatars and graphics that the equipment software own, once analyzed, this data was passed to the spatio-temporal parameters of the software "MarchaHumana ET"

The MarchaHumana ET software determines the spatio-temporal parameters of the human gait guided by the inertial sensors IMU. It presents a report of data such as: distance, step length, step time, step speed, stride length, stride time and walking speed for the prosthetic patient based on the averages of the Technaid® equipment data [4]. The patient to be studied retained a transtibial prosthesis (dynamic foot, pin suspension). This patient is $170 \mathrm{~cm}$ tall and weighs $80 \mathrm{~kg}$. 42 years old with 11 years of amputation.

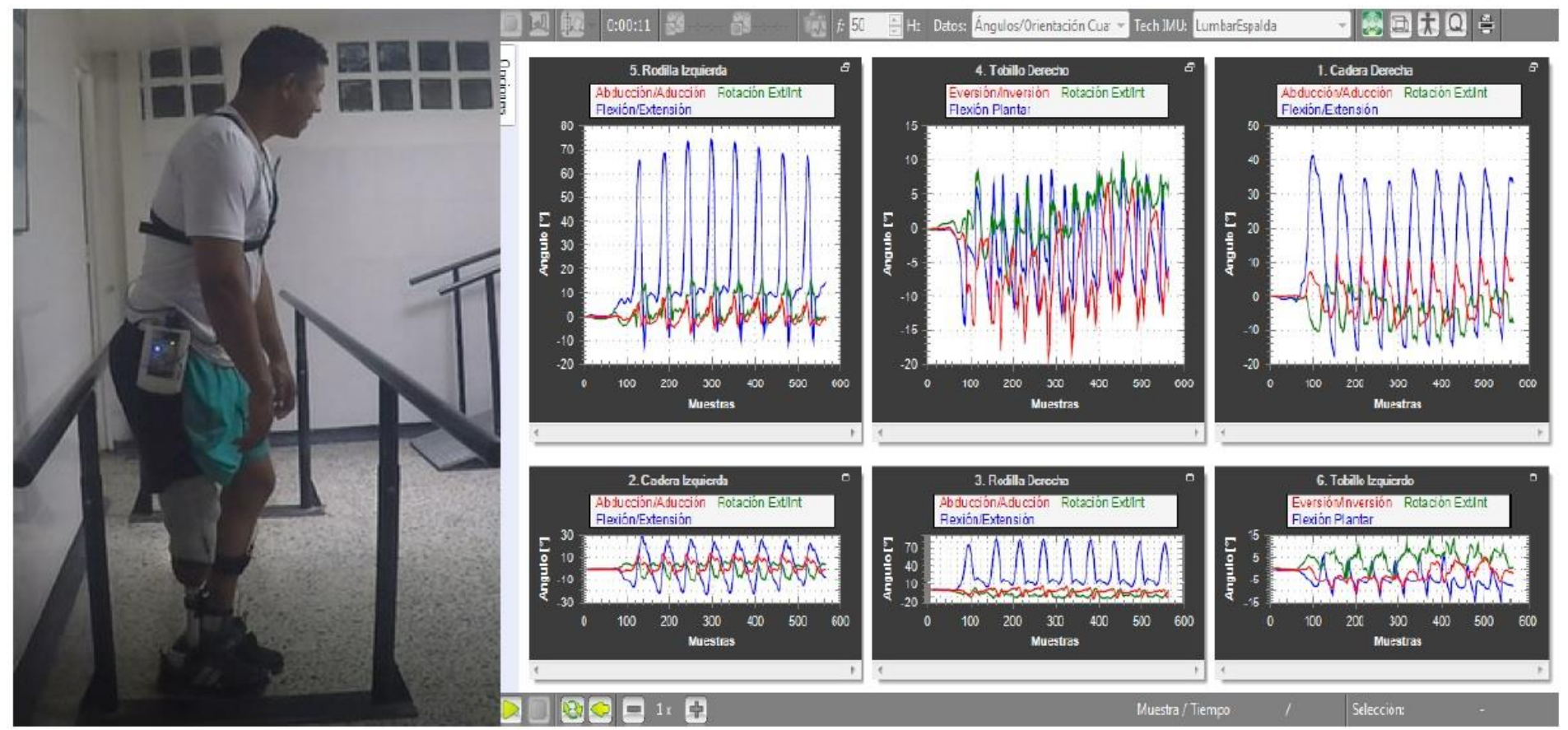

Fig. 1: Sagittal Plane of the Protestive Patient. Graph of angles Vs. Samples of Capture processes, equipment Technaid®.

\section{Results}

To carry out the respective analysis, the extreme data from the report of the spatio-temporal parameters of the software "MarchaHumana ET" were obtained in order to find possible adverse data and to compare them in an atypical way. For the study, these samples were averaged.

Table 1: General parameters of the spatio-temporal variables[5].

\begin{tabular}{|l|l|l|l|}
\hline $\begin{array}{l}\text { Spatio-temporal } \\
\text { parameter }\end{array}$ & Age & Author & Magnitude \\
\hline $\begin{array}{l}\text { Spontaneous } \\
\text { speed (m/s). }\end{array}$ & $18-49$ & Whittle & $1.10-1.82$ \\
\hline Step length (m) & $19-59$ & Van Uden & 0.77 \\
\hline Stride Time (s) & $19-59$ & Van Uden & 1.10 \\
\hline Cadence of step & $18-49$ & Whittle & $91-135$ \\
\hline & $22-40$ & Menz & 110.7 \\
\hline
\end{tabular}


Table 2: Extreme spatio-temporal variables of the aligned socket.

\begin{tabular}{|l|l|}
\hline & ALIGNMENT \\
\hline Step length $(\mathrm{m})$ & 0.283 \\
\hline $\begin{array}{l}\text { Cadence of step } \\
\text { (steps / m) }\end{array}$ & 51 \\
\hline Stride length $(\mathrm{cm})$ & 56.62 \\
\hline Stride time $(\mathrm{s})$ & 2.42 \\
\hline Gait speed $(\mathrm{m} / \mathrm{s})$ & 0.2483 \\
\hline
\end{tabular}

The first step was to take a data report when the patient was in normal alignment. Table 2 shows that for the prosthetic patient, his passage length is $0.283 \mathrm{~m}$ which indicates that it is a very small magnitude when compared to a healthy patient, on the other hand the velocity in comparison with general parameters (Table 1) show that it makes the gait cycle in a very slow way which guarantees that the time and the cadence are of very small magnitudes.

Table 3: Extreme spatio-temporal variables in abduction and adduction for each angular modification of the socket.

\begin{tabular}{|l|l|l|}
\hline & ABDUCTION & ADDUCTION \\
\hline & \multicolumn{2}{|c|}{ 6 degrees } \\
\hline Step length (m) & 0.248 & 0.228 \\
\hline Cadence of step (steps / m) & 34.44 & 22.26 \\
\hline Stride length (cm) & 49.7 & 45.6 \\
\hline Stride time (s) & 3.1 & 2.4 \\
\hline Gait speed (m / s) & 0.145 & 0.82 \\
\hline
\end{tabular}

After studying the patient in his normal alignment conditions, it is proceed to misalign the socket at $6^{\circ}$ and in this way taking the data for joint movements. In abduction and adduction, according to table 3 it was found that, compared to the aligned socket, the length of the stride is very close, as the time. The greatest difference found is in the cadence of the steps then at the time of abduction when the leg moves away from the reference line is faster, generating the greatest magnitude, the opposite happened with the adduction movement. At the moment of adduction at $6^{\circ}$ misalignment, it is evident that the data tend to decrease, evidencing that the higher the degrees of modification, the data tend to increase and in this way resemble a patient's parameters according to the general parameters (Table 1).

Table 4: Extreme spatio-temporal variables in flexion and extension for each angular modification of the socket.

\begin{tabular}{|l|l|l|}
\hline & FLEXION & EXTENSION \\
\hline & 2 degrees & 6 degrees \\
\hline Step length $(\mathrm{m})$ & 0.238 & 0.264 \\
\hline $\begin{array}{l}\text { Cadence of step } \\
\text { (steps / m) }\end{array}$ & 46.92 & 54 \\
\hline Stride length $(\mathrm{cm})$ & 47.6 & 52.9 \\
\hline Stride time $(\mathrm{s})$ & 3.1 & 2.9 \\
\hline Gait speed $(\mathrm{m} / \mathrm{s})$ & 0.187 & 0.255 \\
\hline
\end{tabular}

However, for the flexion and extension movements the data were taken for a misalignment of $2^{\circ}$ to $6^{\circ}$ respectively. According to Table 4 the data show that at $2^{\circ}$ the result is closer to the average and the larger degrees tend to move away from the average, in addition the time remains the same as during the movement of abduction with the difference that the gait speed increases, the step length and the cadence of step increase. In the flexion movement at $6^{\circ}$ it is evident that it is the angle where there is more speed, also the step length is the one of greater magnitude, besides the cadence of step is half of the average. 


\section{Discussion}

When performing the analysis of the gait cycle in the patient, it was observed that it is indispensable to have a suitable space for the movement, ensuring that the patient is comfortable and performs the movement in the most natural way.

A patient with good conditions was found, experiencing 11 years in such circumstances, he was observed physically well, since he leads an active life, when performing activities that demand physical exercise and muscular movement, besides, he was mentally willing to observations and with a good personality, which implies that the limitation was mental and not physical.

On the other hand, it is important to know the opinion of the specialist because carrying a diagnosis for a long time of the state of the amputated person; he knows in what conditions the prosthesis should be, as well, its alignment. Since, in this case, his prosthesis is suspension per pin which indicates that the alignment of the prosthesis diverges according to the context in which it is and therefore is in constant modification.

Finally it is evident how a person in amputation condition is accustomed to handle and assimilate the malfuntion of the alignment and misalignment of the prosthesis and to assume movements as unequal steps, exaggeration of the movement of the prosthesis, to make greater standing force in one of his two joints, etc. in order to be able to move.

\section{Conclusion}

It is concluded that the prosthesis supplies the basic needs of the patient such as displacement and mobility of all parts of the joint, but does not guarantee that his movement and the gait cycle are carried out correctly. In first instance, when analyzing the data in condition of the aligned socket, its parameters diverge compared to the general parameters. For the misalignment conditions, its parameters are similar to the aligned socket but slightly more adverse, guarantee that, regardless of alignment and modifications, the prosthesis is not in suitable conditions for a normal gait cycle. As evidenced, the correct thing is to increase the misalignment angles so that the data is assimilated to a normal pattern.

\section{Acknowledgements}

We thank "Universidad Distrital Francisco Jose de Caldas" for its accompaniment in the studies carried out, the research group DIGITI attached to the University for the valuable study monitoring and access to the used devices in the research. The patient and the specialist are also thanked for their time and willingness. Finally, the orthopedic and rehabilitation center for providing its spaces to acquire the measurements of the patient.

\section{References}

[1] S. Hincapie, D. Munoz, "Physiotherapeutic Approach to Diagnosis: Gait Analysis," Revista Facultad de Ciencias Salud, University of Cauca., vol. 12.

[2] ADDRESS AGAINST MINES, [Online]. Available: http://www.accioncontraminas.gov.co/Paginas/AICMA.aspx

[3] TECHNAID, Tech IMU CV. 4 - Technical specifications, [Online]. Available: http://www.technaid.com/support/documentation

[4] L. Yara, J. Sanches, "Identification of the spatiotemporal variables of the human march from Tech MCS," pp. 30-40, 2016.

[5] J. Camara, "Analysis of the march: its phases and spatio-temporal variables," vol. 7, no. 1, pp. 160-173, ISSN 19003803, 2011, [Online]. Available: http://www.scielo.org.co/scielo.php?script=sci_arttext\&pid=S190038032011000100011\&lng=en\&nrm=iso.

[6] L. Daza, "Clinical - Functional evaluation of the human body," Editorial Medical Panamericana, vol. 1, no. 1, pp. 27-47.

[7] C. Diaz Novo, N. Lopez Rios, A. Montoya Pedron, H. Carvajal Fals, "Preliminary assessment of gait in healthy individuals," vol. 11, no. 44 pp. 135-140, 2007, [Online]. Available: http://www.scielo.org.ve/scielo.php?script=sci_arttext\&pid=S1316-48212007000300005\&lng=en\&nrm=iso 